\title{
Multi Modality Imaging for Cardiac Masses Can Avoid Unnecessary Surgical Intervention: A Case Report of Caseous Calcification of Mitral Annulus
}

\author{
Karam Alzuhairi*, Tomas Zaremba, Eva Korup \\ Department of Cardiology, Aalborg University Hospital, Aalborg, Denmark. \\ Email: *karam.majeed@rn.dk \\ Received November $8^{\text {th }}, 2012$; revised December $19^{\text {th }}, 2012$; accepted January $9^{\text {th }}, 2013$
}

\begin{abstract}
Mitral Annular Calcification (MAC) is a degenerative process involving the fibrous ring of the mitral valve. Caseous Calcification of Mitral Annulus (CCMA) is a rare variant of MAC. This condition is benign and usually needs no treatment. This is a case report of a 67-year-old woman with non-specific symptoms in whom a cardiac tumor was suspected by transthoracic Echocardiography. The use of multi-modality imaging strategy with transesophageal Echocardiography and cardiac MR scanning revealed however the diagnosis of CCMA and spared the patient an unnecessary operation.
\end{abstract}

Keywords: Caseous Calcification of Mitral Annulus; Cardiac Tumors; Cardiac MR

\section{Introduction}

Mitral Annular Calcification (MAC) is a degenerative process involving the fibrous ring of the mitral valve. It represents deposition of calcium in the space between the posterior left ventricular wall and the posterior leaflet of the mitral valve. MAC is found in $10 \%$ of patients older than 50 years of age [1]. Caseous Calcification of Mitral Annulus (CCMA) is a rare variant of MAC. It consists of a mixture of calcium, fatty acids and cholesterol. CCMA has a prevalence of $0.6 \%$ in patients with MAC [1-3].

CCMA is a benign process that carries a good prognosis [4]. It requires usually no surgical treatment unless it causes severe mitral valve regurgitation or embolic complication. Because CCMA does not normally require intervention, it is important to exclude other differential diagnoses that might do so, such as cardiac myxoma, abscess or thrombus.

The authors present a case of a 67-year-old woman with CCMA.

\section{Case Report}

Sixty seven years old woman with a history of diabetes mellitus, arterial hypertension, hypothyroidism and dyslipidemia, was referred to our institution due to suspected myxoma in the left atrium. The patient suffered 2 episodes of a sudden onset fatigue and sweating, in which

*Corresponding author. the patient had to sit down. The episodes lasted only a few minutes and there was no loss of consciousness. During a one-day observation at the local hospital after the first episode, physical examination, ECG and blood tests including coronary markers where within normal range. The patient remained asymptomatic until two months later. After the second episode the patient was referred to a private cardiologist, who performed a transthorasic echocardiography (TTE). A myxoma in the left atrium was suspected and the patient was referred to our institution for further assessment.

The physical examination and ECG were unremarkable.

The TTE (Figure 1) and the transoesophageal echocardiography (TEE) revealed a calcified process attached to the mitral posterior ring. No mitral regurgitation was found. There was a good function of the left ventricle and no other valve disease was present

The mass was suspected to be a caseous calcification of the mitral annulus.

The patient was then referred to Cardiac Magnetic Resonance (CMR) scanning which confirmed a single $24 \times$ $21 \times 20 \mathrm{~mm}$ homogenous mass located at the posterior aspect of the mitral ring. The function of the mitral valve did not seem to be significantly affected at Steady State Free Precession (SSFP) cine. Compared to myocardium, the tumor was hypointense on SSFP (Figure 2), double inversion recovery (IR) T1, triple IR T1 and triple IR T2 
fast spin echo sequences. On early post-contrast SSFP (early gadolinium enhancement), the mass was hypointense as well. IR gradient echo (late gadolinium enhancement) revealed only a thin hyperenhanced rim surrounding the tumor (Figure 3). No extracardiac involvement or pericardial effusion was present. The CMR supported the diagnosis of CCMA.

Due to the benign diagnosis and that symptoms considered unlikely to be related to the condition, no indication was found for surgical treatment. The patient was scheduled for an out-patient TTE follow up after 1 year.

\section{Discussion}

The differential diagnosis of cardiac masses is very important, as the treatment and prognosis can vary widely. CCMA has a favorable prognosis compared to cardiac tumors or an abscess [4].

Primary cardiac tumors are rare, with an estimated incidence of $0.002 \%-0.2 \%$ from surgical series [5,6]. Approximately $75 \%$ are benign with nearly $50 \%$ of those being myxomas [7]. Myxomas most frequently occur between the third and sixth decade and are more common in women. They most commonly originate in the left atrium (75\%), $15 \%-20 \%$ in the right atrium, and the remaining originate in the ventricles. Typically, myxomas are attached by a short pedicle to the border of the fossa ovalis. Clinical features include embolisation (30\% - $40 \%$ of patients), cardiac obstructive symptoms, and constitutional symptoms [8]. On echocardiography myxoma typically represents a mobile cluster arising from the left atrial side of the interatrial septum attached with a stalk. Nevertheless, up to $10 \%$ of myxomas are sessile and lack a stalk [9]. CMR of a myxoma typically reveals a mass arising from the interatrial septum on a short pedicle. It demonstrates isointense Signal Intensity (SI)

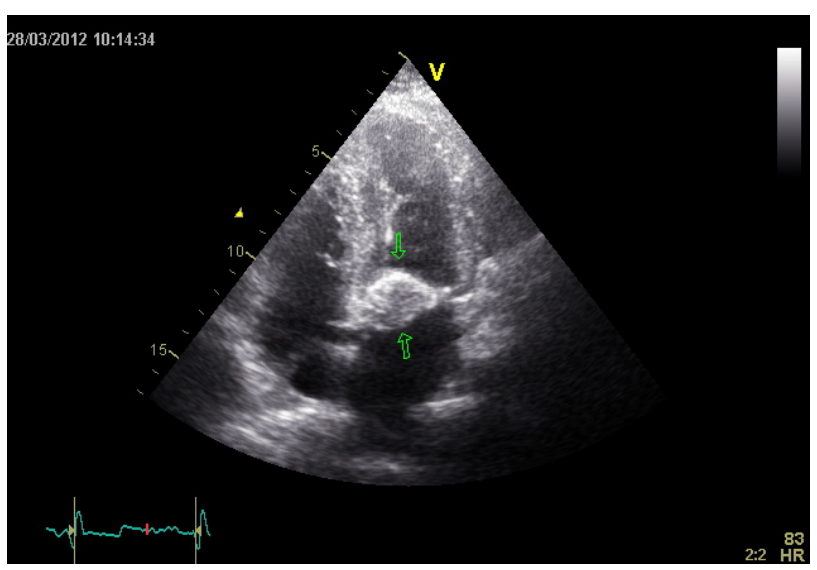

Figure 1. A four chamber view of tranthoracic echocardiography showing a calcified process attached to the mitral valve ring.

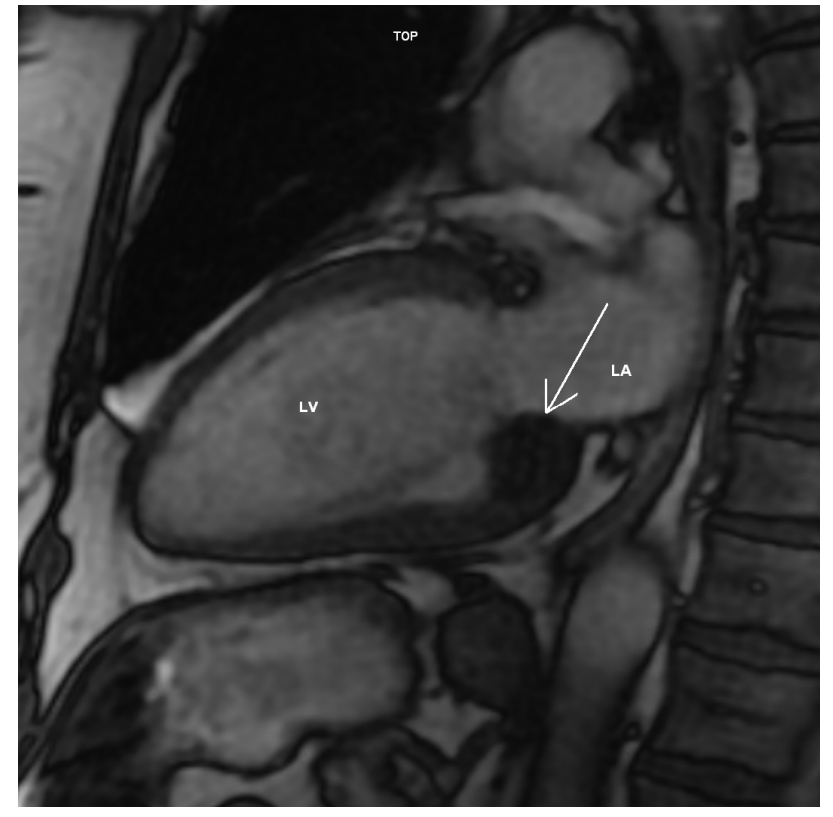

Figure 2. Steady state free precession 2-chamber image at end-diastole showing a hypointense (relative to myocardium) mass at the posterior aspect of the mitral annulus (arrow). LA-left atrium. LV-left ventricle.

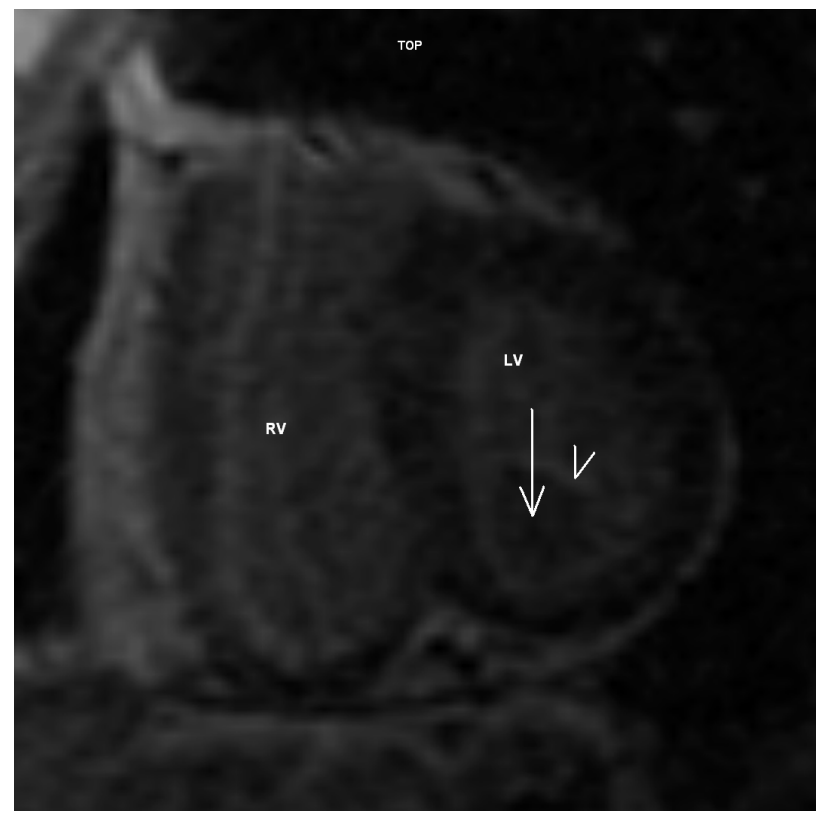

Figure 3. Inversion recovery gradient echo short-axis image showing a mass without late gadolinium enhancement (arrow) surrounded by a thin enhancing rim (arrowhead). LV -left ventricle. $R V$-right ventricle.

on T1-weighted sequences with predominantly high signal on T2-weighted images. Contrast-enhanced T1-weighted images show marked heterogeneous enhancement of the mass. SSFP sequences demonstrate the mass to be mobile [10]. Due to their vascularity, myxomas typically 
perfuse with first-pass gadolinium contrast and enhance to variable extent on delayed enhancement imaging. Myxomas can be distinguished from benign tumors such as lipomas and fibromas using T2-weighted imaging; both lipoma and fibromas are typically dark on T2 weighted images compared with the T2 enhancement characteristic of myxoma [11].

Thrombus is sometimes difficult to differentiate from a myxoma by echocardiography, but it can be done using CMR [12]. A thrombus on CMR would not be expected to display enhancement following administration of contrast media, although chronic thrombi can occasionally accumulate gadolinium peripherally if there is a fibrinous pseudocapsule. [13].

A cardiac abscess is also an important diagnosis to take into consideration, as it is defined with echocardiography as a "mass within the myocardium or annular region with a nonhomogenous echogenic or echolucent appearance." [14], but in our case there is no clinical suspicion of an active endocarditis using the Duke criteria [15].

CCMA is a rare variant of MAC. Eventual symptoms are not different from those of MAC such as palpitations, syncope, and symptoms of mitral regurgitation. Common clinical characteristics in patients with CCMA are: female gender $(72 \%-74 \%)$, mean age of 65 years, hypertension (47\% - 100\%), diabetes ( $21 \%$ - 35\%), palpitation and dyspnea (up to $40 \%$ ) as the most common symptoms, and mitral regurgitation $(67 \%$ - 100\%) $[1,3,16]$. Some of these clinical characteristics apply for our patient. A typical echocardiographic presentation of CCMA is, as in this case, a round, calcified, echodense mass, fixedly attached to the posterior mitral annulus with distinct borders and an echolucent center. Typically there would be no flow noted in the center of the mass by color Doppler $[1,3,17]$.CMR reveals a mass between the posterior mitral annulus and adjacent myocardium, with no enhancement directly after contrast administration and demonstrating peripheral late gadolinium enhancement $[18,19]$.

The use of echocardiography as a standard imaging method in the investigation of suspected cardiac masses is advised as it is cheap, available and does not imply a risk of radiation to the patient, but in cases where the consequence of the diagnosis is an operation, and the diagnosis is not certain as myxoma can rarely presented as a calcified mass [20], the diagnosis should be confirmed using another imaging modality such as CMR, as echocardiography alone can lead to an operation on a wrong diagnosis. [16].

As mentioned above, CCMA has a favorable prognosis and surgery should only be performed in the case of complications such as severe dysfunction of the mitral valve or embolic event. Even the possibility of self re- gression of CCMA has been mentioned in the literatures $[21,22]$.

\section{Conclusion}

CCMA is a rare presentation of MAC, and should be considered as an important differential diagnosis of cardiac masses involving the mitral annulus, as it is a benign process, typically not requiring surgical treatment. We advocate multi modality imaging strategy in suspected CCMA to confirm the diagnosis and to avoid unnecessary surgical intervention.

\section{REFERENCES}

[1] D. Harpaz, I. Auerbach, Z. Vered, M. Motro, A. Tobar and S. Rosenblatt, "Caseous Calcification of the Mitral Annulus: A Neglected, Unrecognized Diagnosis," Journal of the American Society of Echocardiography, Vol. 14, No. 8, 2001, pp. 825-831.

[2] I. Kronzon, H. E. Winer and M. L. Cohen, "Sterile, Caseous Mitral Anular Abscess," Journal of the American College of Cardiology, Vol. 2, No. 1, 1983, pp. 186-190. doi:10.1016/S0735-1097(83)80391-X

[3] G. Deluca, M. Correale, R. Ieva, et al., "The Incidence and Clinical Course of Caseous Calcification of the Mitral Annulus: A Prospective Echocardiographic Study,” Journal of the American Society of Echocardiography, Vol. 21, No. 7, 2008, pp. 828-833. doi:10.1016/j.echo.2007.12.004

[4] K. Teja, R. S. Gibson and S. P. Nolan, “Atrial Extension of Mitral Annular Calcification Mimicking Intracardiac Tumor," Clinical Cardiology, 1987; Vol. 10, No. 9, pp. 546-548. doi:10.1002/clc.4960100918

[5] P. Centofanti, E. Di Rosa, L. Deorsola, et al., "Primary Cardiac Neoplasms: Early and Late Results of Surgical Treatment in 91 Patients," The Annals of Thoracic Surgery, 1999; Vol. 68, No. 4, pp. 1236-1241. doi:10.1016/S0003-4975(99)00700-6

[6] A. W. Elbardissi, J. A. Dearani, R. C. Daly, et al., "Survival after Resection of Primary Cardiac Tumors: A 48Year Experience,” Circulation, Vol. 118, No. 14, 2008, pp. S7-S15. doi:10.1161/CIRCULATIONAHA.107.783126

[7] A. Burke, J. Jeudy Jr and R. Virmani, "Cardiac Tumours: An Update,” Heart, Vol. 94, No. 1, 2008, pp. 117-123. doi:10.1136/hrt.2005.078576

[8] K. Reynen, “Cardiac Myxomas,” The New England Journal of Medicine, Vol. 333, No. 24, 1995, pp. 1610-1617. doi:10.1056/NEJM199512143332407

[9] M. G. St John Sutton, L. A. Mercier, E. R. Giuliani, et al. "Atrial Myxomas: A Review of Clinical Experience in 40 Patients,” Mayo Clinic Proceedings, Vol. 55, No. 6, 1980, pp. 371-376.

[10] C. A. Ridge, R. P. Killeen, K. M. Sheehan, et al., "Giant Right Atrial Myxoma: Characterization with Cardiac Magnetic Resonance Imaging,” Clinical Imaging, Vol. 34, No. 
3, 2010, pp. 231-233.

doi:10.1016/j.clinimag.2009.06.027

[11] C. M. Kramer and W. G. Hundley, "Atlas of Cardiovascular Magnetic Resonance Imaging: An Imaging Companion to Braunwald's Heart Disease,” 1st Edition, Saunders, Philadelphia, 2010.

[12] J. F. Heitner, I. Klem, K. Alexander, et al., "The Case of the Disappearing Myxoma,” Journal of Cardiovascular Magnetic Resonance, Vol. 7, No. 5, 2005, pp. 841-843. doi:10.1080/10976640500288156

[13] J. Vogel-Claussen, C. E. Rochitte, K. C. Wu, et al., "Delayed Enhancement MR Imaging: Utility in Myocardial Assessment," RadioGraphics, Vol. 26, No. 3, 2006, pp. 795-810. doi:10.1148/rg.263055047

[14] Y. L. Kwak and J. W. Shim, “Assessment of Endocarditis and Intracardiac Masses by TEE,” International Anesthesiology Clinics, Vol. 46, No. 2, 2008, pp. 105-120. doi:10.1097/AIA.0b013e31815d13ab

[15] D. T. Durack, A. S. Lukes and D. K. Bright, "New Criteria for Diagnosis of Infective Endocarditis: Utilization of Specific Echocardiographic Findings,” American Journal of Medicine, Vol. 96, No. 3, 1994, pp. 200-209. doi:10.1016/0002-9343(94)90143-0

[16] N. P. McKernan, W. C. Culp Jr, W. L. Knight, W. E. Johnston, R. M. Sniecinski and H. L. Lazar, "CASE 2-2012 Intraoperative Diagnosis and Management of Caseous Calcification of the Mitral Annulus," Journal of Cardiothoracic and Vascular Anesthesia, Vol. 26, No. 2, 2012, pp. 327-332. doi:10.1053/j.jvca.2011.07.015
[17] G. Minardi, C. Manzara, G. Pulignano, et al., "Caseous Calcification of the Mitral Annulus with Mitral Regurgitation and Impairment of Functional Capacity: A Case Report,” Journal of Medical Case Reports, Vol. 2, No. 1, 2008, p. 205. doi:10.1186/1752-1947-2-205

[18] G. Di Bella, P. G. Masci, J. Ganame, et al., "Liquefaction Necrosis of Mitral Annulus Calcification: Detection and Characterization with Cardiac Magnetic Resonance Imaging,” Circulation, Vol. 117, No. 12, 2008, pp. e292e294. doi:10.1161/CIRCULATIONAHA.107.729905

[19] S. Ribeiro, A. Salgado, N. Salomé, et al., "Caseous Calcification of the Mitral Annulus: A Multi-Modality Imaging Perspective,” Revista Portuguesa de Cardiologia, Vol. 31, No. 4, 2012, pp. 313-316. doi:10.1016/j.repc.2012.02.007

[20] S. K. Singh, A. Kumar, V. Tewarson, et al., "Calcified Left Atrial Myxoma with Osseous Metaplasia,” Indian Journal of Chest Disease and Allied Science, Vol. 54, No. 3, 2012, pp. 201-203.

[21] S. Gramenzi, A. A. Mazzola, B. Tagliaferri, et al., "Caseous Calcification of the Mitral Annulus: Unusual Case of Spontaneous Resolution,” Echocardiography, Vol. 22, No. 6, 2005, pp. 510-513. doi:10.1111/j.1540-8175.2005.04056.x

[22] N. Yokoyama, K. Komo, S. Suzuki, et al., "Serial Assessment of Liquefaction Necrosis of Mitral Annulus Calcification by Echocardiography and Multislice Computed Tomography," Circulation, Vol. 115, No. 1, 2007, pp. e1e2. doi:10.1161/CIRCULATIONAHA.106.635599 